(c) The Author(s), 2022. Published by Cambridge University Press on behalf of The Nutrition Society. This is an Open Access article, distributed under the terms of the Creative Commons Attribution licence (http://creativecommons.org/licenses/by/4.0/), which permits unrestricted re-use, distribution and reproduction, provided the original article is properly cited.

\title{
Relationship between the fasting status during hospitalisation, the length of hospital stay and the outcome
}

\author{
Yuka Muto ${ }^{1 \dagger}$, Ayano Kurosawa ${ }^{1 \dagger}$, Chieri Ukita ${ }^{2}$, Norio Hanafusa ${ }^{3}$ and Satoru Nagata ${ }^{4 *}$ \\ ${ }^{1}$ Division of Clinical Nutrition, Tokyo Women's Medical University, Shinjuku-ku, Tokyo, Japan \\ ${ }^{2}$ Division of Clinical Nutrition, International University of Health and Welfare, Narita-shi, Chiba, Japan \\ ${ }^{3}$ Department of Hemodialysis and Apheresis, Tokyo Women's Medical University, Shinjuku-ku, Tokyo, Japan \\ ${ }^{4}$ Department of Paediatrics, Tokyo Women's Medical University, Shinjuku-ku, Tokyo, Japan
}

(Submitted 22 December 2021 - Final revision received 17 February 2022 - Accepted 18 February 2022 - First published online 23 February 2022)

\section{Abstract}

The effects of long-term fasting on the prognosis and hospital economy of hospitalised patient have not been established. To clarify the effects of long-term fasting on the prognosis and hospital economy of hospitalised patients, we conducted a prospective observational study on the length of hospital stay of patients hospitalised at thrity-one private university hospitals in Japan. We conducted a prospective observational study on the effects of fasting period length on the length of hospital stay and outcome of patients hospitalised for 3 months in those hospitals. Of the 14172 cases of hospitalised patients during the target period on the reference day, 770 cases (median 71 years old) were eligible to fast for the study. The length of hospital stay for fasting patients was 33 (4-387) days, which was about 2.4 times longer than the average length of hospital stay for all patients. A comparative study showed the length of hospital stay was significantly longer in the long-term-fasting (fasting period $>10 \mathrm{~d} ; n 386$ ) group than in the medium-term-fasting ( $<10 \mathrm{~d} ; n 384)$ group (median $21 \mathrm{v} .50 ; P<0.0001)$. Although the discharge to home rate was significantly higher in the medium-term-fasting group $(71.4 \% v .36 .5 \% ; P<0.0001)$, the mortality rate was significantly higher in the long-term fasting group $(10 \cdot 8 \% v .25 \cdot 8 \% ; P<0.0001)$. It was verified that the longer the fasting period during hospitalisation, the longer the length of hospital stay and lower home discharge rate, thus indicating that patient quality of life and hospital economy may be seriously dameged.

\section{Key words: Fasting period: Hospital stay: Home discharge rate: Mortality: Private university hospitals}

Several studies have reported that nutritional status during hospitalisation is closely related to treatment efficacy, length of stay and mortality ${ }^{(1-4)}$.

Although it is desirable to take meals orally, as a nutrition management method during hospitalisation, enteral nutrition and intravenous nutrition may be selected, depending on the disease or treatment method. As a rule, the intestinal tract is generally used when the intestinal tract is functioning, and it is considered clinically possible that intestinal mucosal homeostasis is maintained by the administration of nutrients into the intestinal tract, resulting in the maintenance of mechanical and immunological barrier functions ${ }^{(5,6)}$. Furthermore, it has been reported that starting enteral nutrition early reduces the incidence of infectious complications and improves the prognosis ${ }^{(7-11)}$. Conversely, there have been no clear clinical studies as of yet on the superiority of enteral nutrition over intravenous nutrition ${ }^{(12-15)}$.

Therefore, there is a tendency for the judgement of fasting or the period of fasting, along with nutritional management during the fasting period, to be different among facilities and among medical departments. To clarify the effects of long-term fasting on the prognosis and hospital economy of hospitalised patients, we investigated changes in BMI, haematological parameters, administered nutrients, components, etc., to examine the relationship between the inpatient fasting period, length of stay and outcome.

To demonstrate that proper nutritional management of inpatients leads to a better prognosis for patients, ultimately bringing financial benefits to hospitals, the effects of the duration of inpatient fasting on the length of hospital stay and outcome were investigated. The primary outcome was set to verify that the longer the fasting period, the longer the length of hospital stay, while the secondary outcome was to verify that the longer the fasting period, the lower the weight, the lower the haematological parameters and the lower the discharge to home rate.

\section{Materials and methods}

Ethics

This study was a multi-institutional joint study among private university hospitals in Japan and was implemented in accordance with World Medical Association's Declaration of Helsinki

*Corresponding author: Satoru Nagata, email nagata.satoru@twmu.ac.jp

These authors contributed equally to this work. 
upon receiving approval from TWMU Ethics Committee, Approval Number 4963-R2 followed by the ethics review committees of each institution. Informed consent was not obtained from each patient because as per the ethics committee of the university it was unnecessary but opt-out methods in this study.

\section{Target patients}

The subjects consisted of patients who had fasted for at least ten consecutive meals, before and after dinner on the reference date (a specific day in July 2017), and patients aged 1 or older who had consumed three meals a day, among the inpatients at the study target facilities. Fasting was defined as the absence of using the gastrointestinal tract for oral intake, nasal nutrition, gastrostomy, etc.

\section{Target facilities}

Of the eighty facilities registered with the Japan Private Medical Universities Nutrition Study Group, thirty one facilities ( $611 \pm 385$ approved hospital beds) cooperated in the study. The number of dietitians was $9.2 \pm 7.9$ per facility. $93 \%$ of facilities had operating Nutrition Support Team (NST), while $56 \%$ of facilities were determined to have added a NST, $72 \%$ had ward charge systems for supervising dietitians and $14 \%$ had registered dietitians stationed in the ward.

\section{Observation period}

The observation period was set from the hospitalisation date to the observation end date ( 3 months after the reference date). For patients who continued hospitalisation after the observation end date, the observation end date was set as the date of clinical outcome and observation was discontinued. Outcomes were categorised into five types: hospitalised; discharged to home; discharged to a nursing home (discharge to facilities); transferred and died.

\section{Information collection method}

At each target facility, the survey items of the study subjects were input and collected in unified Microsoft Office Excel 2013.

\section{Survey item}

We investigated the target patients' age, sex, medical department in charge (medical department), medical reasons for fasting (reasons for fasting), length of hospital stay, days from admission to the start of fasting, fasting days and the days from the end of fasting to the outcome. The nutritional administration status during the fasting period was investigated based on the nutritional administration method (no administration, peripheral venous nutrition, central venous nutrition (including use with peripheral venous nutrition)), energy administration per day, ratio of administered energy to basal metabolism (energy administration/basal metabolism), administered protein mass and use or non-use of fat preparations. Biochemical findings included BMI, serum Alb $^{(16,17)}$ and peripheral blood $\mathrm{Hb}$, at the time admission and outcome ${ }^{(18-20)}$.

\section{Statistical analysis}

The results of the survey items were described as the mean ( \pm standard deviation) for variables that were normally distributed among quantitative variables, and the median (minimum-maximum) for variables that were not normally distributed. Less than $5 \%$ was considered statistically significant, in this test, using a twogroup comparison with the Wilcoxon signed rank test, $t$ test and $\mathrm{X}^{2}$ test. With respect to the survival analysis, a Cox proportional hazards model was used. Covariates were adjusted for age, sex, medical department, reason for fasting, BMI at admission, Alb at admission, $\mathrm{Hb}$ at admission, nutritional administration method, administered energy/basal metabolic rate and the use or non-use of fat preparations.

Propensity score matching was performed to match the patient background of fasting patients. The matching items were age, sex, BMI at admission, Alb at admission and $\mathrm{Hb}$ at admission. The overall treatment variables were applied to the patients who had fasted less than $10 \mathrm{~d}$ (hereinafter, medium-term fasting group) and patients who had fasted for $10 \mathrm{~d}$ or more (hereinafter, long-term fasting group). The effects on hospitalisation days and outcomes, depending on the fasting period, were analysed using the propensity score calculated from the logistic regression model. Survival time was analysed as a log rank test and sensitivity analysis, while death was defined as a competitive event, in this test, and analysed using Gray's test. The Cochran-MantelHaenszel test was performed for stratified analysis in propensity score matching. JMP Pro 14.0.0 and SAS University Edition were used for the analysis.

\section{Results}

\section{Overview of fasting patients}

The number of patients who were provided meals on the reference day was 12 462, while the number of patients who were instructed to fast was $1710.770(5.4 \%)$ of these patients had fasted for more than ten meals.

The median age was 71 years and included 474 men (61.6\%) and 296 women (38.4\%). The median length of stay among fasting patients was $33 \mathrm{~d}$ (4-387), approximately 2.4 times longer than the average length of stay of $13.9 \mathrm{~d}$ among all hospitalised patients.

\section{Outcomes of fasting patients}

The discharge to home rate was $53.8 \%$, whereas the transfer rate was $19 \cdot 8 \%$, the mortality rate was $18.4 \%$ and $8 \%$ continued hospitalisation on the end day of observation.

\section{Comparison of medium-term and long-term fasting groups}

Because the median fasting period of fasting patients was $10 \mathrm{~d}$, the medium-term (3-10 d fasting period: $n$ 384) group was defined as the medium-term fasting group, while the long-term (fasting period 11-194 d: $n$ 386) group was defined as the longterm fasting group for comparison. There was no significant 
Table 1. Comparison of fasting patient groups

\begin{tabular}{|c|c|c|c|c|c|}
\hline \multirow{2}{*}{$\frac{\text { Characteristics }}{n}$} & \multicolumn{2}{|c|}{$\begin{array}{l}\text { Medium-term fasting } \\
\text { group }\end{array}$} & \multicolumn{2}{|c|}{ Long-term fasting group } & $P$ \\
\hline & 384 & & 386 & & - \\
\hline Age (years) & 71 & $2-111$ & 70 & $1-98$ & - \\
\hline Sex $(M: F)$ & 233:151 & & $241: 145$ & & - \\
\hline Fasting days (days) & $5 \cdot 7$ & $3 \cdot 3-9 \cdot 7$ & 23.3 & $10-193 \cdot 7$ & $<0.0001$ \\
\hline Length of hospital stay (days) & 21 & $4-184$ & 50 & $12-387$ & $<0.0001$ \\
\hline Hospitalisation-fasting start (days) & 1 & $0-175$ & 1 & $0-291$ & 0.0006 \\
\hline Reason for fasting: Surgery-related (\%) & 34.4 & & $12 \cdot 7$ & & $<0.0001$ \\
\hline Gastrointestinal tract rest (gastrointestinal bleeding, etc.) (\%) & 31.5 & & $35 \cdot 0$ & & - \\
\hline End of fasting-outcome (days) & 10 & 0-89 & 8 & 0-89 & 0.0006 \\
\hline BMI change rate (\%: Hospitalisation-at the time of outcome) & -3.4 & $-31-21$ & $-5 \cdot 8$ & $-34-23$ & 0.0014 \\
\hline Serum Alb change rate (\%: Hospitalisation-at the time of outcome) & $-9 \cdot 4$ & $-65-118$ & $-14 \cdot 3$ & $-76-113$ & 0.0012 \\
\hline Blood $\mathrm{Hb}$ change rate (\%: Hospitalisation-at the time of outcome) & $-5 \cdot 3$ & $-64-159$ & -8.4 & $-52-159$ & 0.0038 \\
\hline Amount of energy administration $(\mathrm{kcal} / \mathrm{d})$ & 473 & 0-2017 & 623 & $0-1989$ & $<0.0001$ \\
\hline Administered protein $(\mathrm{g} / \mathrm{d})$ & 39 & $0-77$ & 22.9 & $0-69$ & $<0.0001$ \\
\hline Fat formulation usage $(\%)$ & 5.5 & & $33 \cdot 2$ & & $<0.0001$ \\
\hline Total parenteral nutrition usage rate $(\%)$ & $12 \cdot 2$ & & 63.0 & & $<0.0001$ \\
\hline Home discharge rate $(\%)$ & $71 \cdot 4$ & & $36 \cdot 8$ & & $<0.0001$ \\
\hline Mortality (\%) & 10.9 & & $25 \cdot 8$ & & $<0.0001$ \\
\hline
\end{tabular}

difference between the two groups in terms of age and sex (Table 1).

Fasting reason. The reason why 'intestinal tract was unavailable' was related to 'surgery' in $35 \%$ of patients in the medium-term fasting group and $13 \%$ in the long-term fasting group. The most common reason that surgery was not related was 'gastrointestinal dysfunction due to the underlying disease,' with $32 \%$ in the medium-term fasting group and $35 \%$ in the longterm fasting group.

Energy intake. Although the average daily energy administration was significantly higher in the long-term fasting group than in the medium-term fasting group $(P<0.0001)$, neither group reached basal metabolism. The average daily protein administration and the usage rate of fat preparations were significantly higher in the long-term fasting group than in the medium-term fasting group $(P<0 \cdot 0001$, respectively).

Nutrition methods. The use of central venous nutrition was significantly higher in the long-term fasting group than in the medium-term fasting group $(12.2 \% v .62 .9 \%: P<0.0001)$.

Nutrition evaluation. The long-term fasting group had significantly lower figures in terms of BMI, serum Alb value and peripheral blood Hb value (BMI: $P=0 \cdot 0014$, serum Alb: $P=0 \cdot 0012$ ), peripheral blood $\mathrm{Hb}: P=0.0038$ ), respectively, at the time of outcome than admission, compared with the medium-term fasting group.

Length of hospital stay. The length of hospital stay was significantly longer in the long-term fasting group than in the mediumterm fasting group $(P<0.0001)$.

\section{Outcome}

The discharge to home rate was significantly higher in the medium-term fasting group than in the long-term fasting group
(71.4\% v. 36.5\%, $(P<0 \cdot 0001))$. Conversely, mortality was significantly higher in the long-term fasting group than in the medium-term fasting group $(10.8 \% v .25 .8 \%, P<0.0001)$.

The hazard ratio between the two groups was calculated to demonstrate that items other than fasting days (such as age and nutritional status at admission) did not significantly affect the probability of being discharged. The results indicated hazard ratio, 3.34; $95 \%$ CI, 2.55, $4.38(P<0.0001)$ when covariates were considered, and hazard ratio, 3.82; $95 \%$ CI, 2.94, 4.97 $(P<0 \cdot 0001)$ after propensity score matching (Table 2$)$.

The medium-term fasting group was associated with higher discharge rates than the long-term fasting group in all subgroups (Fig. 1). Furthermore, the survival time analysis at competitive risk events, using the data after propensity score matching, indicated that the cumulative discharge rate was significantly higher in the medium-term fasting group than in the long-term fasting group (Fig. 2; $P<0.0001$ )

As a result of the survival time analysis for competitive risk events, using the data after propensity score matching, the cumulative discharge rate was significantly higher in the medium-term fasting group than in the long-term fasting group.

\section{Discussion}

In Japan, due to the benefits of the universal health insurance system, the burden on patients for hospitalization costs is low and the length of hospital stay tends to be longer than in other countries $^{(21)}$. Moreover, the use of intravenous nutrition formulas in Japan is higher than in other countries because much of the expenses are covered by health insurance ${ }^{(22)}$.

However, prolonged use of intravenous nutrition can lead to the extinction of gastrointestinal function and immunity, resulting in reduced patient Quality of Life (QOL) and wasted hospital expenses due to bloodstream infections and complications ${ }^{(23-27)}$.

Because it is difficult to clarify the income and expenditure situation at each hospital, the calculation of 'number of hospital stays' can be cited as a powerful tool that reflects this. 
Table 2. Cox comparison of probability of discharge using proportional hazard model

\begin{tabular}{llcccr}
\hline Parameters & Group & Number of people & Hazard ratio & $95 \%$ confidence interval & $P$ \\
\hline \multirow{2}{*}{ No adjustment } & Medium-term fasting group & 386 & - & - & - \\
& Long-term fasting group & 384 & 4.1 & $3.32,5.03$ & - \\
Covariate* $^{*}$ & Medium-term fasting group & 314 & - & - & - \\
Propensity score matching $^{* *}$ & Long-term fasting group & 313 & 3.4 & $2.55,4.38$ & $<0.0001$ \\
& Medium-term fasting group & 231 & - & $2.94,4.97$ & $<0.0001$ \\
& Long-term fasting group & 231 & 3.8 &
\end{tabular}

* Covariates included age, sex, BMl at admission, Alb at admission, $\mathrm{Hb}$ at admission, presence or absence of fat preparations, nutritional administration method and energy dose/ basal metabolism.

${ }^{* \star}$ The matching items were age, sex, BMl at admission, Alb at admission and $\mathrm{Hb}$ at admission.

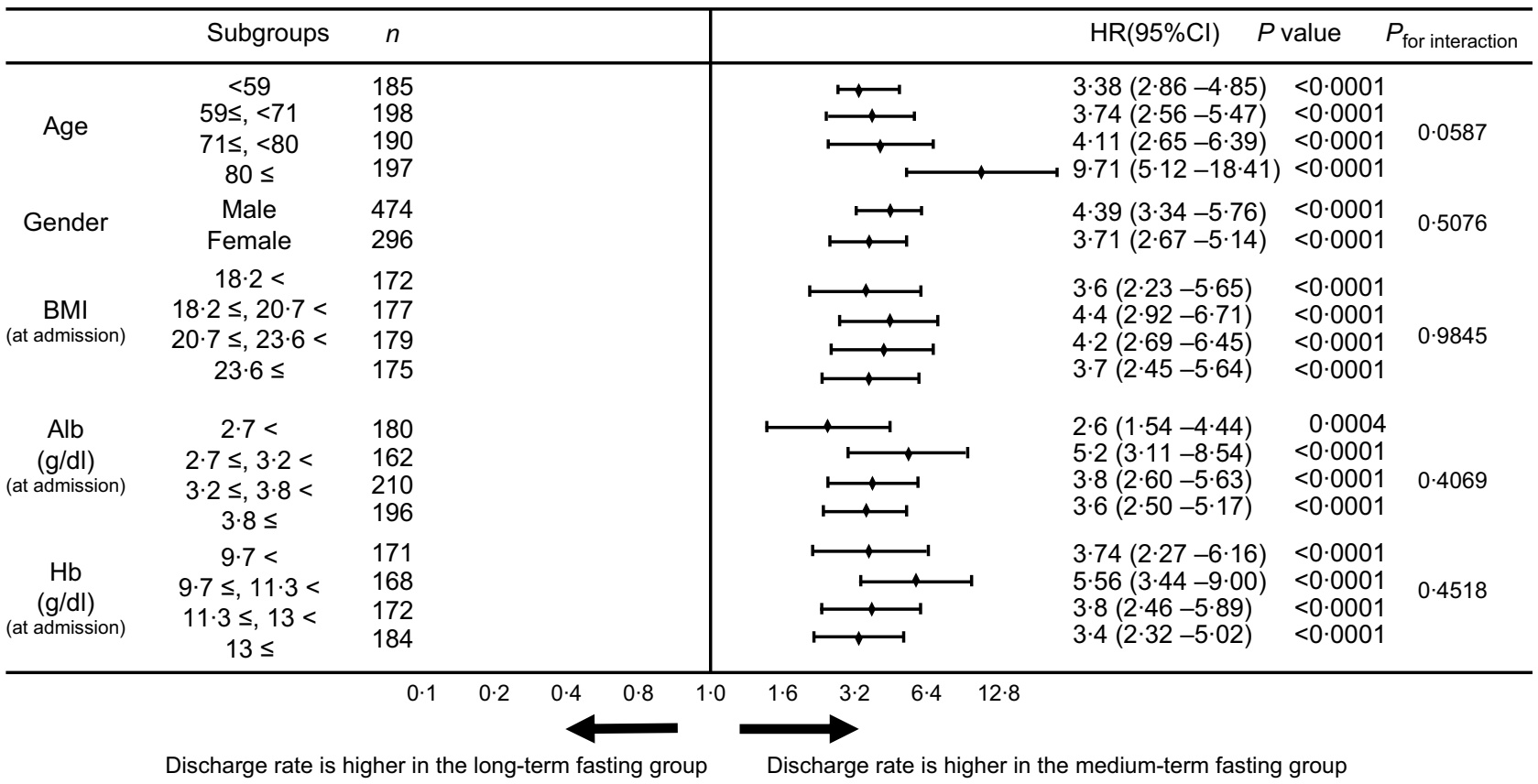

Fig. 1. Comparison of probability of discharge from hospital using hazard ratios of BMI and haematological parameters.

Because private hospitals affiliated with private universities in Japan are of a certain size and tend to have similar business conditions, the length of hospital stay is generally constant, making it suitable for estimating the impact of certain conditions on the hospital economy. Here, the authors focus on patient discharge rates and hospital stays as a measure of the impact of patient fasting on QOL and hospital profits. To demonstrate that the fasting period can severely damage the nutrition of patients, we examined changes in BMI and blood parameters.

As a result, the length of hospital stay for fasting patients was more than 2.4 times longer than the average length of hospital stay at the targeted private university hospitals. To find out if the longer the fasting period, the longer the hospital stay, a comparison was made between the medium-term fasting group with a fasting period of less than $10 \mathrm{~d}$ and the long-term fasting group with more than $10 \mathrm{~d}$, the results of which indicated that the length of hospital stay was significantly extended in the latter. Therefore, the possibility was suggested that the longer the fasting period, the lower the hospital's profit, in Japan.

Next, we investigated whether the fasting period during hospitalization affects the discharge to home rate of patients and it was verified that prolonging the fasting period significantly reduces the patient discharge to home rates and furthermore increase mortality. Furthermore, it has been reported that intervention by a dietitian has contributed to the improvement of the nutritional status of patients ${ }^{(28-30)}$. Going forward, we would like to examine the relationship between the active intervention of dietitians, the length of stay among patients and the discharge to home rates.

Lastly, to clarify that the reason why longer fasting periods lead to longer lengths of hospital stays and clearly the deteriorated nutritional status of patients in hospital, we compared changes in BMI, serum Alb and peripheral blood $\mathrm{Hb}$, that are haematological parameters, at the time of outcomes among medium-term and long-term fasting groups. As a result, it was revealed that the longer the fasting period, the significantly lower the BMI, serum Alb level, and peripheral blood $\mathrm{Hb}$ level at the time of outcome, compared with the time of admission, and that fasting clearly worsens the nutritional status of the patient. It was also highlighted that patients were discharged from hospital with poor nutrition. It should be noted here that intravenous energy administration, the amount of administered protein and the 


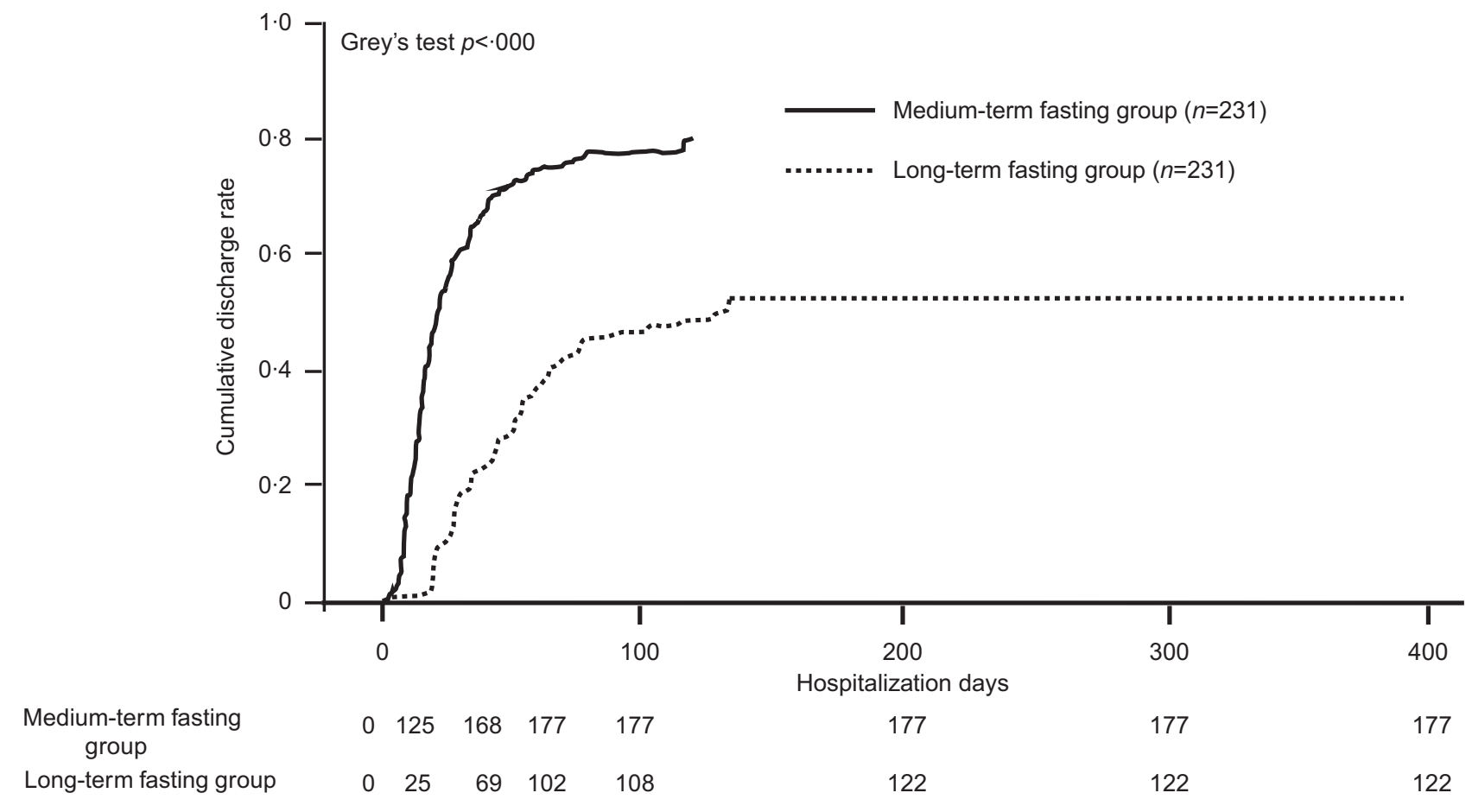

Fig. 2. Survival time analysis for competing risk events.

amount of fat were significantly higher in the long-term fasting group than in the medium-term fasting group. In other words, no matter how much the nutritionally correct amount of energy is calculated and components are contrived, there is little benefit to patients and hospitals in providing them intravenously. The reason for this is that nutrition through the gastrointestinal tract is most physiological in humans, helping to assure homeostasis of metabolism and immune activity, as verified by a variety of evidence. However, it is difficult to verify that this theory is correct, even in actual clinical settings, with this study being the first to prove it with a sufficient number of samples, as far as we know ${ }^{(31)}$.

As this study was all conducted by registered dietitians at private university hospitals in Japan, the authority to conduct the survey was limited. With respect to the haematological parameters, the ESPEN provides evidence for nutritional evaluation, the use of the CONUT score (consisting of serum Alb value, peripheral blood total lymphocyte counts and serum total cholesterol value), which provided evidence for nutritional evaluation with ESPEN, could have provided a simpler study with more evidence. However, the preliminary surveys indicated that routine measurements of total cholesterol levels at each facility were low and had to be excluded from this survey.

This study was associated with some limitations. First of all, since this study was a simple observational study, comparisons could not be carried out regarding various key items such as the length of hospital stay under the same background conditions, such as the severity of disease in the fasting and non-fasting groups. In addition, it was not possible to exclude the possibility that the fasting group may have been affected by a group with serious diseases compared to the non-fasting group. Going forward, we plan to collaborate with the NST of all hospitals to assess the severity of the fasting group and non-fasting group and to also investigate the length of hospital stay, BMI and CONUT score, using a prospective study design.

\section{Conclusion}

As a result of the investigation on the effect of the duration of fasting by hospitalised patients, on the length of hospital stay and outcome, it has been verified that the longer the fasting period during hospitalisation, the longer the length of hospital stay, weight loss, decreased haematological parameters and lower home discharge rates.

\section{Acknowledgements}

We would like to offer special thanks to our colleagues (the facilities) for cooperating with the research as follows: Akiko Ono (Iwate Medical University Hanamaki Onsen Hospital), Yoshiko Sakai (Nippon Medical School Hospital), Mariko Sameda (Toho University Sakura Medical Center), Noriko Terakado (Tokyo Medical University Ibaraki Medical Center), Shoko Fukaya (Tokyo Medical University Hachioji Medical Center), Hisako Yoshida (The Jikei University Katsushika Medical Center), Ai Yuasa (The Jikei University Kashiwa Hospital), Miyuki Shimazui (Showa University Northen Yokohama Hospital), Smith Eriko (Kansai Medical University Kori Hospital), Akemi Katayama (Osaka Medical College Hospital), Misako Ando (Kurume University Medical Center), Teruko Sato (Kitasato University Hospital), Mariko Sanada (Kitasato University Hospital), Satsuki Hata (Kitasato University Medical Center), Yoshie Tsukada (Kyorin University Hospital), Yoko Endo (Kawasaki Medical University Hospital), Miyori Sasano (Kawasaki Medical University General Medical 
Center), Hitomi Asakura (Teikyo University Hospital), Minae Mogami (Teikyo University Chiba Medical Center), Eiji Ebiko (Fujita Health University Banbuntane Hotokukai Hospital), Kazue Araki (Hyogo College of Medicine College Hospital), Koji Mino (Hyogo College of Medicine Sasayama Medical Center), Yoko Motojima (Saitama Medical Center, Saitama Medical University), Katsue Saito (Dokkyo Medical University Hospital), Tamotsu Aso (Dokkyo Medical University Nikko Medical Center), Katsumi Kajiwara (Kindai University Hospital), Hiroyo Miyata (Kindai University Sakai Hospital), Mami Sugano (Kindai University Nara Hospital), Yoko Goto (Tokai University Tokyo Hospital) and Noriko Ota (Wakamatsu Hospital of the University of Occupational and Environmental Health).

Conceptualization, S.N.; methodology, C.U.,N.H. and S.N.; validation, S.N.; formal analysis, A.K., Y.M.,C.U.,N,H. and S.N.; investigation, A.K. and Y.M.; data curation, A.K., Y.M., N.H. and S.N.; writing-original draft preparation, A.K. and Y.M.; writing-review and editing, S.N.; visualization, A.K., Y.M. and S.N.; supervision, S.N.; project administration, A.K., Y.M., C.U. and S.N. All authors have read and agreed to the published version of the manuscript.

This research received no specific grant from any funding agency, commercial, or not-for-profit sectors.

The authors declare that there is no conflict of interest.

\section{References}

1. Lewis SJ, Egger M, Sylvester PA, et al. (2001) Early enteral feeding $v$. 'nil by mouth' after gastrointestinal surgery: systematic review and meta-analysis of controlled trials. BMJ 323, 773-776.

2. Lewis SJ, Andersen HK \& Thomas S (2009) Early enteral nutrition within $24 \mathrm{~h}$ of intestinal surgery $v$. later commencement of feeding: a systematic review and meta-analysis. J Gastrointest Surg 13, 569-575.

3. Lim SL, Ong KC, Chan YH, et al. (2012) Malnutrition and its impact on cost of hospitalization, length of stay, readmission and 3-year mortality. Clin Nutr 31, 345-350.

4. Holst M, Yifter-Lindgren E, Surowiak M, et al. (2013) Nutritional screening and risk factors in elderly hospitalized patients: association to clinical outcome? Scand J Caring Sci 27, 953-961.

5. Wilmore DW, Smith RJ, O'Dwyer ST, et al. (1988) The gut: a central organ after surgical stress. Surgery 104, 917-923.

6. Hernandez G, Velasco N, Wainstein C, et al. (1999) Gut mucosal atrophy after a short enteral fasting period in critically ill patients. J Crit Care 14, 73-77.

7. Moore EE \& Jones TN (1986) Benefits of immediate jejunostomy feeding after major abdominal trauma - a prospective, randomized study. J Trauma 26, 874-881.

8. Reynolds JV, O'Farrelly C, Feighery C, et al. (1996) Impaired gut barrier function in malnourished patients. Br J Surg $\mathbf{8 3}$, 1288-1291.

9. Chappell VL, Thompson MD, Jeschke MG, et al. (2003) Effects of incremental starvation on gut mucosa. Dig Dis Sci 48, 765-769.

10. Andersen HK, Lewis SJ \& Thomas S (2006) Early enteral nutrition within $24 \mathrm{~h}$ of colorectal surgery $v$. later commencement of feeding for postoperative complications. Cochrane Database Syst Rev 2006, Cd004080.

11. Haac B, Henry S, Diaz J, et al. (2018) Early enteral nutrition is associated with reduced morbidity in critically ill soft tissue patients. Am Surg 84, 1003-1009.
12. Windsor AC, Kanwar S, Li AG, et al. (1998) Compared with parenteral nutrition, enteral feeding attenuates the acute phase response and improves disease severity in acute pancreatitis. Gut 42, 431-435.

13. Jeejeebhoy KN (2001) Total parenteral nutrition: potion or poison? Am J Clin Nutr 74, 160-163.

14. Pacelli F, Bossola M, Papa V, et al. (2001) Enteral $v$. parenteral nutrition after major abdominal surgery: an even match. Arch Surg 136, 933-936.

15. Seike J, Tangoku A, Yuasa Y, et al. (2011) The effect of nutritional support on the immune function in the acute postoperative period after esophageal cancer surgery: total parenteral nutrition $v$. enteral nutrition. $J$ Med Invest 58, 75-80.

16. Kondrup J, Allison SP, Elia M, et al. (2003) ESPEN guidelines for nutrition screening 2002. Clin Nutr 22, 415-421.

17. Hamada Y (2015) Objective Data Assessment (ODA) methods as nutritional assessment tools. J Med Invest 62, 119-122.

18. Dong X, Mendes de Leon C, Artz A, et al. (2008) A populationbased study of hemoglobin, race, and mortality in elderly persons. J Gerontol A Biol Sci Med Sci 63, 873-878.

19. Riva E, Tettamanti M, Mosconi P, et al. (2009) Association of mild anemia with hospitalization and mortality in the elderly: the Health and Anemia population-based study. Haematologica 94, 22-28.

20. Chalmers KA, Knuiman MW, Divitini ML, et al. (2012) Longterm mortality risks associated with mild anaemia in older persons: the Busselton Health Study. Age Ageing 41, 759-764.

21. Kato N, Kondo M, Okubo I, et al. (2014) Length of hospital stay in Japan 1971-2008: hospital ownership and cost-containment policies. Health Policy 115, 180-188.

22. nutritionDay (2016) nutritionDay November 2015 in JAPAN. https://www.jspen.or.jp/wp-content/uploads/2018/03/ND15_ country_report_JP_en-1.pdf (accessed October 2021).

23. Turpin RS, Canada T, Rosenthal V, et al. (2012) Bloodstream infections associated with parenteral nutrition preparation methods in the United States: a retrospective, large database analysis. JPEN J Parenter Enteral Nutr 36, 169-176.

24. Abunnaja S, Cuviello A \& Sanchez JA (2013) Enteral and parenteral nutrition in the perioperative period: state of the art. Nutrients 5, 608-623.

25. Doig GS, Chevrou-Severac H \& Simpson F (2013) Early enteral nutrition in critical illness: a full economic analysis using US costs. Clinicoecon Outcome Res 5, 429-436.

26. Reintam Blaser A, Starkopf J, Alhazzani W, et al. (2017) Early enteral nutrition in critically ill patients: ESICM clinical practice guidelines. Intensive Care Med 43, 380-398.

27. Pradelli L, Graf S, Pichard C, et al. (2018) Supplemental parenteral nutrition in intensive care patients: a cost saving strategy. Clin Nutr 37, 573-579.

28. Soguel L, Revelly JP, Schaller MD, et al. (2012) Energy deficit and length of hospital stay can be reduced by a two-step quality improvement of nutrition therapy: the intensive care unit dietitian can make the difference. Crit Care Med 40, 412-419.

29. Belanger V, McCarthy A, Marcil V, et al. (2019) Assessment of malnutrition risk in canadian pediatric hospitals: a multicenter prospective cohort study. J Pediatr 205, 160-167.e6.

30. Schuetz P, Fehr R, Baechli V, et al. (2019) Individualised nutritional support in medical inpatients at nutritional risk: a randomised clinical trial. Lancet 393, 2312-2321.

31. Fuentes Padilla P, Martínez G, Vernooij RW, et al. (2019) Early enteral nutrition (within $48 \mathrm{~h}$ ) $v$. delayed enteral nutrition (after $48 \mathrm{~h}$ ) with or without supplemental parenteral nutrition in critically ill adults. Cochrane Database Syst Rev 2019, CD012340. 